\title{
Ultrasound Technology as a Novel Treatment Strategy in Pancreatic Cancer
}

\section{ISSN: 2637-773X}

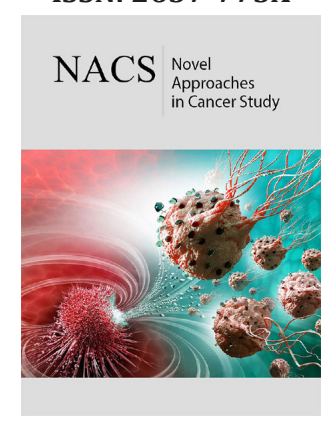

*Corresponding author: Julie Earl, Medical Oncology Department, Ramón y Cajal Health Research Institute (IRYCIS), Carretera Colmenar Km 9,100. 28034. Madrid, Spain,Email: julie.earl@live.co.uk

Submission: 海 June 12, 2019

Published: 海June 17, 2019

Volume 2 - Issue 5

How to cite this article: Iciar G, Antonio $\mathrm{R}$, Luis M R, Alberto P, Luis H. Ultrasound Technology as a Novel Treatment Strategy in Pancreatic Cancer. Nov Appro in Can Study. 2(5). NACS.000550.2019.

DOI: $10.31031 /$ NACS.2019.02.000550

Copyright@ Julie Earl, This article is distributed under the terms of the Creative Commons Attribution 4.0 International License, which permits unrestricted use and redistribution provided that the original author and source are credited.
Iciar González Gómez ${ }^{1}$, Antonio Ramos Fernández , Luis M Rodríguez-Lorenzo $^{2}$, Alberto Pinto del Corral ${ }^{1}$, Luis Hernández ${ }^{1}$, Jesús Frutos Díaz-Alejo ${ }^{3}$, Vanessa Pachón Olmos ${ }^{4}$, Cristian Perna ${ }^{5}$ and Julie Earl ${ }^{3,6 *}$

${ }^{1}$ Institute of Physical and Information Technologies (ITEFI), CSIC, Madrid, Spain

${ }^{2}$ Institute of Science and Technology of Polymers (ICTP-CSIC), Madrid, Spain

${ }^{3}$ Molecular Epidemiology and Predictive tumor markers group, Ramón y Cajal Health Research Institute (IRYCIS), Madrid, Spain

${ }^{4}$ Medical Oncology Department, Ramón y Cajal University Hospital, Madrid, Spain

${ }^{5}$ Department of Pathology, Ramón y Cajal Health Research Institute (IRYCIS), Madrid, Spain

${ }^{6}$ Biomedical Research Network in Cancer (CIBERONC), Madrid, Spain

\section{Opinion}

Adenocarcinoma of the pancreas (PDAC) accounts for $2.4 \%$ of all cancers diagnosed and is the fourth leading cause of cancer death, with almost equal rates of incidence and mortality [1]. By 2030, pancreatic cancer is projected to be the second leading cause of cancer-related death [2], surpassing breast, prostate and colorectal cancer. The overall survival at 5 years of around $7.2 \%$ as the majority of patients present with advanced disease at diagnosis. Patients with localized disease are treated with surgery, with or without neoadjuvant chemotherapy/ radiotherapy, followed by adjuvant chemotherapy. The majority (around 80\%) of patients are treated only with chemotherapy as they have an advanced disease. Patients are treated in the first line with gemcitabine-abraxane or Folfirinox and with Naliri plus 5FU in the second line. There have been few clinical advances in PDAC treatment over the last 20 years and chemotherapy is the only treatment option available for the majority of patients. These tumours are also resistant to many targeted therapies such as anti-EGFR therapy like cetuximab [3] due to the presence of a KRAS mutation in the majority of primary tumors. Personalized medicine strategies have not yet been established in pancreatic cancer as in other more common tumour types. Thus, novel anti-tumour strategies are an important clinical need in order to improve survival rates.

PDAC has a desmoplastic stroma that enhances tumorigenesis and tumor progression and reduces the efficacy of chemotherapy that translates to a poor prognosis and treatment resistance [4]. The tumor stroma is a complex structure composed of extracellular matrix (ECM) proteins such as collagens, fibronectin, laminin, glycoproteins, proteoglycans and glycosaminoglycans and cell types such as immune cells, cancer associated fibroblasts, endothelial cells and neuronal cells [5]. Targeting the stroma as a treatment strategy in pancreatic tumors has gained a lot of attention recently. A preliminary study in mice showed that targeting hedgehog signaling resulted in an enhanced delivery of gemcitabine to the primary tumor $[4,6]$. However, this strategy failed in the clinical trial phase as the elimination of the stroma resulted in a more aggressive phenotype and a reduced progression free survival (PFS) and overall survival (OS) [4]. Hyaluronic acid (HA) is a glycosaminoglycan protein and is an important component of the ECM that functions as a physical and contributes to treatment resistance and preliminary results of clinical trials targeting HA have shown promising results [4]. However, novel strategies are urgently required that complement the effect of chemotherapy agents by targeting tumor cells and the stroma simultaneously. Due to the important role of the stroma in tumor progression, this is also an attractive therapeutic target.

Ultrasounds could be an effective technology to prevent tumor progression. Ultrasounds may provide an effective therapeutic strategy, either alone or in combination with standard 
chemotherapy to improve patient outcome. In particular, high intensity focused ultrasounds HIFU are currently used to perform some tumor ablation processes or combined with chemotherapy treatments for the localized drug delivery. High intensity focused ultrasounds have an intensity of more than $5 \mathrm{~W} / \mathrm{cm} 2$ (Miller et al., 2012) and have thermal mechanical and biological effects on tissues (Zhou, 2011). In fact, HIFU are used in China for the treatment of pancreatic cancer as they specifically target the tumor lesions with reduced secondary effects (Zhou, 2014).

However, the effects of the ultrasounds on cells and tissues can be diverse depending on certain parameters associated with their frequency, duty cycle, bandwidth, wave shape, intensity and manner of application. A strategic selection of these parameter determines the ability of the ultrasounds to destroy the tumor stroma or modify its effects. In principle, the risk of cellular and tissue harm decreases with the intensity of the waves applied. As a consequence, the lower the intensity, the lower the risk of cell damage. Low-Intensity Pulsed Ultrasound (LIPUS) is widely used as an imaging tool in medicine at low acoustic intensities $(<3 \mathrm{~W} / \mathrm{cm} 2)$. It is a non-invasive and safe technique used extensively as a diagnostic and therapeutic tool [711]. Different intensities of exposure have been used in previous studies for therapeutic purposes such as healing of bone-fractures or soft-tissue lesions. Doses of up to $2 \mathrm{~W} / \mathrm{cm} 2$ have been used without tissue damage for frequencies ranging between 0.7 and 3.0 MHz. Various studies have reported LIPUS-induced cell growth with proliferation and promotion of multi-lineage differentiation with cell expansion and differentiation in tissue culture, including gingival cells $[12,13]$, periodontal cells $[14,15]$, cementoblastic cells $[16,17]$, chondrocytes $[18,19]$ or mesenchymal stem cells $[20,21]$.

LIPUS may also have a negative effect on cell growth and proliferation when applied at different intensities. The mechanisms governing these LIPUS-induced effects on tissues and cells $[10,11]$ are not well known and need to be investigated. Further studies are needed to decipher the effects of US on biological parameters such as tumor growth and progression, cell migration and cell signaling pathways. Furthermore, the effects of US on the various cell types that form the tumor and the associated stroma such as cancer associated fibroblasts, macrophages and endothelial cells also need to be studied in detail. The development of this technology for use in the clinical practice would require a multidisciplinary approach with biologists, physicists, oncologists and engineers and may also benefit patients with other tumor types. Frequencies close to $1-2 \mathrm{MHz}$ are being tested in recent experiments on healthy tissues and cell cultures to analyze different effects acoustically induced. The development of new ultrasonic devices to study the cell and tissue acoustic response are a technological challenge. In particular, the combination of microfluidics and ultrasounds could be relevant for the study of cell biodynamics and functional effects induced by the ultrasounds. We are currently developing novel devices to study the biological effects of low intensity ultrasounds in various types of tumor models, including bioprinted 3D tumor \& stroma models. Furthermore, we are developing high-resolution ultrasonic methods for a non-invasive early detection of very small physical changes in tissues related with tumor development such as spatial, thermal and elastic properties [22].

\section{Acknowledgement}

We would like to acknowledge the following funding related with the use of ultrasounds as a novel therapeutic strategy: Spanish National Plan project RETOS DPI 2017-90147-R. Intramural call for new research projects for clinical researchers and emerging research groups. IRYCIS. (2018/0240). Iberoamerican Network CYTED-DITECROD-218RT0545.

\section{References}

1. Ferlay J, Soerjomataram I, Dikshit R, Eser S, Mathers C, et al. (2015) Cancer incidence and mortality worldwide: sources, methods and major patterns in GLOBOCAN 2012. Int J Cancer 136(5): E359-E386.

2. Rahib L, Smith BD, Aizenberg R, Rosenzweig AB, Fleshman JM, et al. (2014) Projecting cancer incidence and deaths to 2030: The unexpected burden of thyroid, liver, and pancreas cancers in the united states. Cancer Res 74(11): 2913-2921.

3. Zeitouni D, Pylayeva GY, Der CJ, Bryant KL (2016) KRAS mutant pancreatic cancer: No lone path to an effective treatment. Cancers (Basel) 8(4): $1-22$.

4. Ahn DH, Ramanathan RK (2017) Targeting the stroma in pancreatic cancer. Chin Clin Oncol 6(6): 1-7.

5. Kleeff J, Korc M, Apte M, Vecchia CLa, Johnson CD, et al. (2016) Pancreatic cancer. Nat Publ Gr 2: 1-23.

6. Olive KP, Jacobetz MA, Davidson CJ, Gopinathan A, McIntyre D, et al. (2010) Inhibition of Hedgehog signaling enhances delivery of chemotherapy in a mouse model of pancreatic cancer. Science 324(5933): 1457-1461.

7. Kristiansen TK, Ryaby JP, McCabe J, Frey JJ, Roe LR (1997) Accelerated healing of distal radial fractures with the use of specific, low-intensity ultrasound: A multicenter, prospective, randomized, double-blind, placebo-controlled study. J Bone Jt Surg Am 79(7): 961-973.

8. Jason WB, Mohit B, Abhaya VK (2002) The effect of low-intensity pulsed ultrasound therapy on time to fracture healing: a meta-analysis. Can Med Assoc J 166(4): 3-7.

9. Claes L, Willie B (2007) The enhancement of bone regeneration by ultrasound. Prog Biophys Mol Biol 93(1-3): 384-398.

10. Mundi R, Petis S, Kaloty R, Shetty V, Bhandari M (2009) Low-intensity pulsed ultrasound: Fracture healing. Indian J Orthop 43(2): 132-140.

11. Xin Z, Lin G, Lei H, Lue TF, Guo Y (2016) Clinical applications of low-intensity pulsed ultrasound and its potential role in urology. Transl Androl Urol 5(2): 255-266.

12. El-Bialy T, Alhadlaq A, Wong B, Kucharski C (2014) Ultrasound effect on neural differentiation of gingival stem/progenitor cells. Ann Biomed Eng 42(7): 1406-1412.

13. Shiraishi R, Masaki C, Toshinaga A, Okinaga T, Nishihara T, et al. (2011) The effects of low-intensity pulsed ultrasound exposure on gingival cells. J Periodontol 82(10): 1498-1503.

14. Harle J, Salih V, Mayia F, Knowles JC, Olsen I (2001) Effects of ultrasound on the growth and function of bone and periodontal ligament cells in vitro. Ultrasound Med Biol 27(4): 579-586.

15. Ren L, Yang Z, Song J, Wang Z, Deng F, et al. (2013) Involvement of p38 mapk pathway in low intensity pulsed ultrasound induced osteogenic differentiation of human periodontal ligament cells. Ultrasonics 53(3): 686-690. 
16. Dalla BDA, Tanaka E, Oka H, Yamano E, Kawai N, et al. (2006) Effects of ultrasound on cementoblast metabolism in vitro. Ultrasound Med Biol 32(6): 943-948.

17. Dalla BDA, Tanaka E, Inubushi T, Oka H, Ohta A, et al. (2008) Cementoblast response to low- and high-intensity ultrasound. Arch Oral Biol 53(4): 318-323.

18. Mukai S, Ito H, Nakagawa Y, Akiyama H, Miyamoto M, et al. (2005) Transforming growth factor-beta 1 mediates the effects of low-intensity pulsed ultrasound in chondrocytes. Ultrasound Med Biol 31(12): 1713-1721.

19. Takeuchi R, Ryo A, Komitsu N, Mikuni TY, Fukui A, et al. (2008) Low-intensity pulsed ultrasound activates the phosphatidylinositol 3 kinase/ akt pathway and stimulates the growth of chondrocytes in three-dimensional cultures: A basic science study. Arthritis Res Ther 10(4): R77.
20. Schumann D, Kujat R, Zellner J, Angele MK, Nerlich M, et al. (2006) Treatment of human mesenchymal stem cells with pulsed low intensity ultrasound enhances the chondrogenic phenotype in vitro. Biorheology 43(3,4): 431-443.

21. Angle SR, Sena K, Sumner DR, Virdi AS (2011) Osteogenic differentiation of rat bone marrow stromal cells by various intensities of low-intensity pulsed ultrasound. Ultrasonics 51(3): 281-288.

22. Bazou D, Maimon N, Munn L, Iciar G (2017) Effects of low intensity continuous ultrasound (LICUS) on mouse pancreatic tumor explants. Appl Sci 7(12): 1275-1288. 